\title{
Decoherence of a quantum two-level system by spectral diffusion
}

\author{
Shlomi Matityahu, ${ }^{1}$ Alexander Shnirman, ${ }^{2,3}$ Gerd Schön, ${ }^{4,5}$ and Moshe Schechter ${ }^{1}$ \\ ${ }^{1}$ Department of Physics, Ben-Gurion University of the Negev, Beer Sheva 84105, Israel \\ ${ }^{2}$ Institut für Theorie der Kondensierten Materie, \\ Karlsruhe Institute of Technology, 76131 Karlsruhe, Germany \\ ${ }^{3}$ L. D. Landau Institute for Theoretical Physics RAS, Kosygina street 2, 119334 Moscow, Russia \\ ${ }^{4}$ Institut für Theoretische Festkörperphysik, Karlsruhe Institute of Technology, 76131 Karlsruhe, Germany \\ ${ }^{5}$ Institute of Nanotechnology, Karlsruhe Institute of Technology, D-76344 Eggenstein-Leopoldshafen, Germany
}

(Dated: September 3, 2018)

\begin{abstract}
We study the dephasing of an individual high-frequency tunneling two-level system (TLS) due to its interaction with an ensemble of low-frequency thermal TLSs which are described by the standard tunneling model (STM). We show that the dephasing by the bath of TLSs explains both the dependence of the Ramsey dephasing rate on an externally applied strain as well as its order of magnitude, as observed in a recent experiment [J. Lisenfeld et al.]. However, the theory based on the STM predicts the Hahn-echo protocol to be much more efficient, yielding too low echo dephasing rates, as compared to the experiment. Also the strain dependence of the echo dephasing rate predicted by the STM does not agree with the measured quadratic dependence, which would fit to a high-frequency white noise environment. We suggest that few fast TLSs which are coupled much more strongly to the strain fields than the usual TLSs of the STM give rise to such a white noise. This explains the magnitude and strong fluctuations of the echo dephasing rate observed in the experiment.
\end{abstract}

\section{INTRODUCTION}

The low-temperature physics of amorphous and disordered solids has been a subject of great interest for more than four decades. Below about $1 \mathrm{~K}$ the acoustic and thermodynamic properties of a large variety of glasses are not only qualitatively different compared to their crystalline counterparts, but show a remarkable degree of universality $\underline{\underline{1}} \underline{\underline{3}}$ This universal behavior was explained by the existence of low-energy excitations with two-level structure, known as tunneling two-level systems (TLSs) $\stackrel{4.5}{=}$ In the standard tunneling model (STM), each TLS is characterized by the energy bias $\Delta$ between its two basis states, the tunnel splitting $\Delta_{0}$ between both, and its coupling $\gamma$ to the strain field $\epsilon$. Thus the Hamiltonian of each TLS in the STM is $s^{\underline{4}} \underline{\underline{6}}$

$$
\hat{\mathcal{H}}_{\mathrm{STM}}=\frac{1}{2}\left(\Delta \hat{\tau}_{z}+\Delta_{0} \hat{\tau}_{x}\right)+\gamma \epsilon \hat{\tau}_{z}
$$

where $\hat{\tau}_{x}$ and $\hat{\tau}_{z}$ are the Pauli matrices in the local states basis. The central idea of the STM is that the TLSs are independent and their energy bias and tunnel splitting are randomly distributed, with a universal distribution function $f\left(\Delta, \Delta_{0}\right)=P_{0} / \Delta_{0}$, where $P_{0}$ is a material dependent constant $\underline{\underline{6}}$

The role of the coupling to the strain field [third term in Eq. (11)] is threefold. First, via this channel the TLS couples to phonons, which gives rise to the energy relaxation of the TLS. Second, by applying mechanical stress externally, one can control and manipulate the TLS $\stackrel{17.18}{1}$ Third, this coupling is at least partially responsible for the coupling between the TLSs. Although the STM has originally considered independent TLSs, it is now well established that TLSs interact via elastic (phonon-mediated) or electric (photon-mediated)

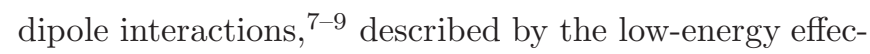
tive Hamiltonian

$$
\hat{\mathcal{H}}_{\mathrm{int}}=\frac{1}{2} \sum_{i \neq j} J_{i j} \hat{\tau}_{z, i} \hat{\tau}_{z, j}
$$

where $\hat{\tau}_{z, i}$ and $\hat{\tau}_{z, j}$ are the pauli matrices that represent the TLSs at sites $i$ and $j$, and the interaction coefficients are

$$
J_{i j}=\frac{c_{i j} U_{i j}}{R_{i j}^{3}}
$$

The parameter $c_{i j} \sim O(1)$ in general is a complicated function of the polar angle between the TLSs at sites $i$ and $j$, but frequently can be treated as a normaldistributed random variable, $R_{i j}$ is the distance between the TLSs, and $U_{i j}$ characterizes the specific interaction. Here we will focus on elastic interactions for which

$$
U_{i j}=\frac{\gamma_{i} \gamma_{j}}{\rho v^{2}}
$$

where $\rho$ and $v$ are the mass density and sound velocity, respectively. Our results can be straightforwardly generalized to the case where electric interactions are present.

With the advent of superconducting quantum bits (qubits), the investigation of the low-temperature properties of amorphous solids has gained further interest 10 for two main reasons. First, ensembles of TLSs are believed to be a major source of fluctuations leading to energy relaxation and decoherence in superconducting qubits and microwave resonators. Specifically, the coherence times of superconducting qubits incorporating Josephson junctions were found to be strongly affected by the presence of TLSs located in the tunnel barriers 
of the junctions $\frac{11,12}{12}$ which are typically made of amorphous aluminum oxide with a thickness of $2-3 \mathrm{~nm}$. A deeper understanding of the nature of TLSs and their role as sources of decoherence is necessary in order to achieve high-fidelity operation of superconducting qubits. Second, it was established that superconducting phase qubits can be utilized to study the properties of individual TLSs $\stackrel{11,13}{=}$ The qubit spectrum exhibits an avoided level crossing at bias values for which the qubit is in resonance with a certain TLS. In addition this resonant coupling allows one to manipulate the quantum state of the TLS directly by coherent single-pulse resonant driving 15 as well as to study the longitudinal $\left(T_{1}\right)$ and transverse $\left(T_{2}\right)$ relaxation times of individual TLSs $\underline{14,15}$

The individual TLSs that are being probed and manipulated by qubits are characterized by the level splitting of the order of that of the qubit $(\sim 2 \pi \cdot 10 \mathrm{GHz})$, which is substantially higher than the temperature in the experiment. To distinguish the (high-frequency, "probed") TLS from, e.g., the TLSs with energy splittings smaller or of the order of the temperature (thermal TLSs), we introduce for the former the subscript $p$ and rewrite its Hamiltonian (11) as 16

$$
\hat{\mathcal{H}}_{p}=\frac{1}{2}\left(\Delta_{p}\left(\epsilon_{p}\right) \hat{\tau}_{z, p}+\Delta_{0, p} \hat{\tau}_{x, p}\right) .
$$

The bias $\Delta_{p}$ is controlled by the applied strain $\epsilon_{p}$ as written in Eq. (11), i.e. $\Delta_{p}\left(\epsilon_{p}\right)=$ const $+\gamma_{p} \epsilon_{p}$. Recently, a static strain tuning of the energy bias of individual TLSs in a superconducting phase qubit was demonstrated, $\underline{17,18}$ The results support the assumption of the STM that the coupling of TLSs to strain fields occurs mainly through a change of the asymmetry bias $\Delta_{p}$, whereas changes of $\Delta_{0, p}$ are negligible.

In Ref. 18 the strain dependence of the relaxation and coherence times of individual high-frequency TLSs was measured, revealing the following interesting features:

(1) The pure dephasing rate $\Gamma_{\varphi, R}$ of the probed TLS, as extracted from the Ramsey (free induction) protocol, is linear with respect to the asymmetry bias $\left|\Delta_{p}\right|$ in the vicinity of the symmetry point $\Delta_{p}=0$. Outside the window $\left|\Delta_{p}\right| \lesssim 2 \pi \cdot 1 \mathrm{GHz}$ around the symmetry point,$\frac{19}{1}$ the Ramsey dephasing rate deviates from the linear behavior, exhibiting small changes of slope or irregularities.

(2) The pure dephasing rate $\Gamma_{\varphi, E}$, as extracted from the Hahn-echo protocol, is much smaller than the Ramsey dephasing rate (with $\Gamma_{\varphi, E} / \Gamma_{\varphi, R} \lesssim 0.1$ at $\Delta_{p} \approx 2 \pi \cdot 1 \mathrm{GHz}$ ), demonstrating the efficiency of the echo protocol in filtering noise at frequencies $\omega \ll 2 \pi \cdot 1 \mathrm{MHz} \sim \Gamma_{\varphi, R}$. Furthermore, the echo dephasing rate grows quadratically with $\Delta_{p}$ in the whole measured range of $\Delta_{p}$. This implies the presence of a white-noise environment inducing energy fluctuations on a time scale $\lesssim 1 \mu$ s.

The starting point of the theoretical description is the following: The individual high-frequency probed TLS with energy splitting $E_{p} \equiv \sqrt{\Delta_{p}^{2}+\Delta_{0, p}^{2}} \gg k_{\mathrm{B}} T$ suffers from decoherence due to its interaction with an ensemble of thermal TLSs with energy splittings lower than the temperature. Such thermal TLSs undergo random transitions (telegraph noise) between their two eigenstates, thereby causing fluctuations in the energy splitting $E_{p}$ of the probed high-frequency TLS. This is the mechanism of spectral diffusion observed in "hole-burning" experiments $\stackrel{20,21}{=}$ As we will show in the following this picture needs to be refined in several directions.

In this paper we provide a detailed theoretical analysis of the coherence properties of an individual highfrequency TLS coupled to an ensemble of thermal TLSs. The qubit used to probe serves merely as a tool and is not part of the modeling. After providing a general theoretical description of the spectral diffusion we analyze the decoherence caused by an ensemble of thermal TLSs with parameters taken from the STM (we call these TLSs " $\tau$-TLSs"). A short description of this theory was provided in Ref. 18. We show that by assuming elastic dipole-dipole interactions as in Eqs. (2)-(41), with a bath of thermal $\tau$-TLSs, one may explain very well both the qualitative dependence on strain as well as the order of magnitude of the Ramsey dephasing rate. This demonstrates that the TLSs introduced in the STM form the main low-frequency noise source in superconducting phase qubits. Moreover, the suggested theory predicts the temperature dependence of the Ramsey dephasing rate (at a fixed strain). For the echo dephasing rate, on the other hand, the theory at this level predicts that the contribution of $\tau$-TLSs is orders of magnitude smaller than the experimental result. In addition, the strain dependence of the echo dephasing rate predicted by the theory is in disagreement with the experiment.

We further show that the experimental results on the echo dephasing rate could be potentially explained by the presence of a few fast thermal TLSs $\underline{\underline{22}}$ These TLSs are characterized by parameters very different from those of the STM ( $\tau$-TLSs). In particular they have a much stronger coupling to the strain, which leads to the enhanced relaxation (switching) rate. From the point of view of the probed TLS the fast thermal fluctuators produce Gaussian noise, since their switching rate is larger than their coupling to the probed TLS. This noise is white at relevant frequencies (frequencies lower than the typical switching rate of the fast thermal TLSs). This explains the quadratic dependence of the echo dephasing rate on the applied strain. Such strongly interacting TLSs (henceforth denoted as $S$-TLSs) were recently introduced within a model suggesting a possible explanation for the universality of acoustic and thermodynamic properties in disordered systems at low temperatures $\underline{23}$ We estimate the order of magnitude for the contribution of the $S$-TLSs to the echo dephasing rate and discuss its relevance in accounting for the experimental findings. We emphasize that in all our considerations we assume the probed TLS to be a standard $(\tau$-)TLS, since these are the prevalent TLSs at low temperatures. 
The paper is organized as follows: in Sec. II we first define the spin-fluctuator model, $24-26$ and then describe its results for the Ramsey and echo decay signals of the probed TLS in different regimes of relaxation rates and TLS-TLS couplings (Sec. IA ). We then discuss the important distinction between average and typical results, which arises in situations in which a quantity is dominated by contributions from a small number of fluctuators (Sec. IIB). The model is applied in Sec. III to the case of an environment consisting of weakly interacting TLSs as postulated in the STM. We first describe the characteristic relaxation rates and TLS-TLS couplings of such TLSs (Sec. IIIA), and then derive the results for the Ramsey (Sec. IIIB) and echo (Sec. IIIC) dephasing rates. In Sec. IV we generalize the model to the model with two types of TLSs introduced in Ref. 23. We first review the two-TLSs model (Sec. IVA), estimate the characteristic relaxation rates and TLS-TLS couplings of strongly interacting $S$-TLSs (Sec. IVB), and then study the contribution of $S$-TLSs to the echo dephasing rate (Sec. IV C). Our conclusions are discussed and summarized in Sec. $\nabla$

\section{THE MODEL}

\section{A. Decoherence}

We consider a single high-frequency probed TLS interacting with a set of thermal TLSs via the Hamiltonian (2). Thus, the Hamiltonian reads

$$
\begin{aligned}
\hat{\mathcal{H}} & =\frac{1}{2}\left(\Delta_{p} \hat{\tau}_{z, p}+\Delta_{0, p} \hat{\tau}_{x, p}\right)+\frac{1}{2} \sum_{j}\left(\Delta_{j} \hat{\tau}_{z, j}+\Delta_{0, j} \hat{\tau}_{x, j}\right) \\
& +\sum_{j} J_{j} \hat{\tau}_{z, p} \hat{\tau}_{z, j}+\hat{\mathcal{H}}_{\text {bath }} .
\end{aligned}
$$

The first term of Eq. (6) describes the probed TLS. The second term describes the set of thermal TLSs. The coupling constants $J_{j}$ denote the coupling strength (3) between the thermal TLS at site $j$ and the probed TLS. The term $\hat{\mathcal{H}}_{\text {bath }}$ describes the internal interactions between the thermal TLSs, as well as their coupling to phonons.

In the eigenbasis of the TLSs, the system is described by the Hamiltonian

$$
\begin{aligned}
\hat{\mathcal{H}} & =\frac{1}{2} E_{p} \hat{\sigma}_{z}+\frac{1}{2} \sum_{j} E_{j} \hat{\alpha}_{z, j}+\frac{1}{2} \hat{\mathcal{X}}\left(\hat{\sigma}_{z} \cos \theta_{p}-\hat{\sigma}_{x} \sin \theta_{p}\right) \\
& +\hat{\mathcal{H}}_{\text {bath }} .
\end{aligned}
$$

Here $\hat{\sigma}_{z}$ are the Pauli matrices in the eigenbasis of the probed TLS and $\hat{\alpha}_{z, j}$ are the Pauli matrices in the eigenbasis of the thermal TLS at site $j$. The first term of Eq. (17) describes the probed TLS with energy $E_{p}=$ $\sqrt{\Delta_{p}^{2}+\Delta_{0, p}^{2}}$ and $\cos \theta_{p}=\Delta_{p} / E_{p}, \sin \theta_{p}=\Delta_{0, p} / E_{p}$. All these are strain-dependent via $\Delta_{p}\left(\epsilon_{p}\right)$. The second term describes the set of thermal TLSs with energies
$E_{j}=\sqrt{\Delta_{j}^{2}+\Delta_{0, j}^{2}} \lesssim T$, and the third term describes the interactions between the probed TLS and the set of thermal TLSs, with the operator $\hat{\mathcal{X}}$ given by

$$
\hat{\mathcal{X}}=2 \sum_{j} J_{j}\left(\hat{\alpha}_{z, j} \cos \theta_{j}-\hat{\alpha}_{x, j} \sin \theta_{j}\right) \text {. }
$$

Here $\cos \theta_{j}=\Delta_{j} / E_{j}$ and $\sin \theta_{j}=\Delta_{0, j} / E_{j}$.

The operator $\hat{\mathcal{X}}$ fluctuates since each thermal TLS, i.e. TLS for which $E_{j}<T$, switches randomly between its two eigenstates. Such random transitions are driven by the emission or absorption of phonons,,$\frac{5,27}{5}$ or by internal interactions between the TLSs $\stackrel{8,28}{ }$ The fluctuations in $\hat{\mathcal{X}}$ add a random contribution to the bare energy $E_{p}$ of the probed TLS through the longitudinal coupling $\left(\propto \hat{\sigma}_{z}\right)$ in the third term of Eq. (77). This results in a dephasing of the probed TLS. Henceforth, we will simplify the model by making the following assumptions:

(1) We consider random telegraph noise of thermally excited TLSs due to relaxation $\left(T_{1}\right)$ processes. TLSs with energy larger than the temperature are frozen in their ground state and are thus irrelevant for the dephasing of the probed TLS. We will thus restrict the sum in Eq. (8) to thermal TLSs. Further, since the probed TLS and the thermal TLSs are non-resonant, we can neglect the transverse term $\left(\propto \hat{\alpha}_{x, j}\right)$ of $\hat{\mathcal{X}}$ in Eq. (8).

(2) We will treat the environment-induced noise classically, i.e., we replace the operator $\hat{\alpha}_{z, j}$ by the classical random variable $\alpha_{z, j}(t)$ (sometimes called a fluctuator) which switches between \pm 1 . Accordingly, $\hat{\mathcal{X}} \rightarrow \mathcal{X}(t)$ is the corresponding sum (8) of such random variables. Such a classical treatment is allowed provided that the frequencies of the random transitions, $\Gamma_{1} \equiv T_{1}^{-1}$, are much smaller than the temperature $T$ and the probed TLS energy splitting $E \underline{29}$

Having modelled the environment of TLSs as a classical noise, we now consider the simplified model

$$
\hat{H}=\frac{1}{2} E_{p} \hat{\sigma}_{z}+\frac{1}{2} X(t) \hat{\sigma}_{z},
$$

with

$$
\begin{aligned}
X(t) & =\cos \theta_{p} \mathcal{X}(t)=2 \cos \theta_{p} \sum_{j} J_{j} \cos \theta_{j} \alpha_{z, j}(t) \\
& =\sum_{j} v_{j} \alpha_{z, j}(t) .
\end{aligned}
$$

Here $v_{j}$ is the effective coupling between the thermal TLS at site $j$ and the probed TLS, given by

$$
v_{j}=2 J_{j} \cos \theta_{j} \cos \theta_{p} \equiv 2 J_{j} \sqrt{1-u_{j}} \cos \theta_{p},
$$

with $u_{j} \equiv \sin ^{2} \theta_{j}=\left(\Delta_{0, j} / E_{j}\right)^{2}$. This model is often re-

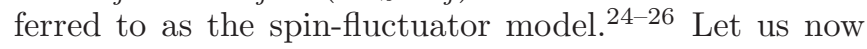




\begin{tabular}{|c|c|c|c|c|}
\hline & \multicolumn{2}{|c|}{$v \gg \Gamma$} & \multicolumn{2}{c|}{$v \ll \Gamma$} \\
\hline & $v t \ll 1$ & $v t \gg 1$ & $\Gamma t \ll 1$ & $\Gamma t \gg 1$ \\
\hline$-\ln f_{R}$ & $\frac{v^{2} t^{2}}{2}$ & $\Gamma t-\ln [\cos (v t)]$ & $\frac{v^{2} t^{2}}{2}$ & $\frac{v^{2}}{\Gamma} t$ \\
\hline$-\ln f_{E}$ & $\frac{v^{2} \Gamma t^{3}}{6}$ & $\Gamma t$ & $\frac{v^{2} \Gamma t^{3}}{6}$ & $\frac{v^{2}}{\Gamma} t$ \\
\hline
\end{tabular}

TABLE I. The different limits of the Ramsey and echo decay signals, Eqs. (14), due to a single fluctuator, characterized by switching rate $\Gamma$ and coupling $v$.

consider the effect of the classical noise (10), which consists of the environment of thermal TLSs, on the Ramsey and echo decay signals $\underline{\underline{30}}$

The decay of a Ramsey signal is given by $f_{R}(t)=$ $\left\langle e^{i \varphi_{R}(t)}\right\rangle$, where $\varphi_{R}(t)$ is the random phase accumulated at time $t$,

$$
\varphi_{R}(t)=-\int_{0}^{t} X\left(t^{\prime}\right) d t^{\prime}
$$

In an echo experiment, the phase acquired is the difference between the two free evolution periods,

$$
\varphi_{E}(t)=-\int_{0}^{t / 2} X\left(t^{\prime}\right) d t^{\prime}+\int_{t / 2}^{t} X\left(t^{\prime}\right) d t^{\prime},
$$

and the corresponding decay function is $f_{E}(t)=$ $\left\langle e^{i \varphi_{E}(t)}\right\rangle$. Since we consider thermal TLSs with $E_{j}<T$, the transition rates in both directions can be assumed to be equal. For a single fluctuator, characterized by switching rate $\Gamma$ and coupling $v$, an average over the switching history yields $\underline{24}-26$

$$
\begin{aligned}
& f_{R}(t)=e^{-\Gamma t}\left(\cos \mu t+\frac{\Gamma}{\mu} \sin \mu t\right), \\
& f_{E}(t)=e^{-\Gamma t}\left[1+\frac{\Gamma}{\mu} \sin \mu t+\frac{\Gamma^{2}}{\mu^{2}}(1-\cos \mu t)\right],
\end{aligned}
$$

where $\mu=\sqrt{v^{2}-\Gamma^{2}}$. The decay function due to a set of fluctuators is the product of individual contributions of the form (14), that is $F_{R / E}(t)=\prod_{j} f_{R / E, j}(t)$.

It is useful to present the main limits of Eqs. (14), as shown in Table I. One can clearly see here the effect of the echo protocol. In the short time limit, $v t \ll 1$ and $\Gamma t \ll 1$, the initial parabolic decay $f_{R}(T) \propto e^{-v^{2} t^{2} / 2}$ is replaced by the much slower decay $f_{E}(t) \propto e^{-v^{2} \Gamma t^{3} / 6}$. In this regime, the decay due to a single fluctuator is small for both Ramsey and echo protocols, but having many fluctuators might make this decay relevant (see below).

For the regime $v>\Gamma$ and $v t \gg 1$ the oscillating entry $\Gamma t-\ln [\cos (v t)]$ requires a separate discussion. These oscillations were discovered in Ref. 25 as an example of a prominent non-gaussian effect. The origin of the oscillations is the interference between two "paths" in which the equally probable \pm state of the fluctuator never changes during a single experimental run. Therefore, these oscillations are almost completely removed by the echo protocol, as can be seen in Table I. If several fluctuators in this regime contribute, the Ramsey decay function will be a product of several incommensurate oscillating functions and, thus, will be strongly suppressed. As we will see below, this is the regime of the Ramsey decay, which occurs at times longer than the typical dephasing times. Finally, in the regime $v<\Gamma$ and $\Gamma t \gg 1$ the echo protocol is inefficient and the result coincides with the one given by the Golden rule (see more details in Sec. IVC).

\section{B. Averaging over the distribution of TLS parameters}

There are three parameters that control the thermal TLSs: i) the energy $E_{j}=\sqrt{\Delta_{j}^{2}+\Delta_{0, j}^{2}}<T$, ii) the normalized tunnel splitting $u_{j} \equiv \sin ^{2} \theta_{j}=\left(\Delta_{0, j} / E_{j}\right)^{2}$, and iii) the distance to the probed TLS $r_{j}$. In a concrete sample at a given strain we have a unique realization of the parameters $\left\{E_{j}, u_{j}, r_{j}\right\}$ characterizing the TLSs, and the total Ramsey/echo decay signal is given by

$$
-\ln F_{R / E}(t)=-\sum_{j} \ln f_{R / E, j}(t) .
$$

We first estimate $\left\langle\ln \left|F_{R / E}(t)\right|\right\rangle_{D}$, where $\langle\ldots\rangle_{D}$ denotes the averaging over the disorder configurations of the TLSs. Since we are dealing with quenched disorder, the question of self-averaging arises and one should examine if the result for $\left\langle\ln \left|F_{R / E}(t)\right|\right\rangle_{D}$ corresponds to a typical situation $\stackrel{31}{=}$ We obtain

$$
\begin{aligned}
& \left\langle\ln \left|F_{R / E}(t)\right|\right\rangle_{D} \\
& =\int d \Omega \int_{R_{0}}^{\infty} r^{D-1} d r \int_{u_{\min }}^{1} d u \int_{0}^{T} d E P(E, u) \ln \left|f_{R / E}\right|
\end{aligned}
$$

where $D$ is the spatial dimension of the amorphous tunnel barrier and

$$
P(E, u)=\frac{P_{0}}{2 u \sqrt{1-u}}
$$

is derived from the standard tunnelling model distribution function $P\left(\Delta, \Delta_{0}\right)=P_{0} / \Delta_{0}$ using the relations $E \equiv \sqrt{\Delta^{2}+\Delta_{0}^{2}}$ and $u \equiv \sin ^{2} \theta=\left(\Delta_{0} / E\right)^{2}$ (see Ref. 32). In Eq. (16) $f_{R / E}=f_{R / E}(v, \Gamma)$ are given by Eqs. (14) and $u_{\min }$ is a lower cutoff for the parameter $u$. It should be emphasized that $\Gamma(E, u)$ depends on $E$ and $u$ [see Eq. (22)], whereas $v(r, u)$ depends on $r$ and $u$ [see Eq. (11)].

According to Table I it is convenient to divide the integration into three domains: a) $\Gamma t<1$ and $v t<1$; b) $v>\Gamma$ and $v t>1$; c) $\Gamma>v$ and $\Gamma t>1$, as illustrated in Fig. 1 Thus, for the Ramsey decay signal we obtain 


$$
\begin{aligned}
-\left\langle\ln \left|F_{R}(t)\right|\right\rangle_{D} & \approx \int d \Omega \int_{R_{0}}^{\infty} r^{D-1} d r \int_{u_{\min }}^{1} d u \int_{0}^{T} d E P(E, u) \theta(1-v t) \theta(1-\Gamma t) \frac{v^{2} t^{2}}{2} \\
& +\int d \Omega \int_{R_{0}}^{\infty} r^{D-1} d r \int_{u_{\min }}^{1} d u \int_{0}^{T} d E P(E, u) \theta(v t-1) \theta(v-\Gamma)[\Gamma t-\ln |\cos (v t)|] \\
& +\int d \Omega \int_{R_{0}}^{\infty} r^{D-1} d r \int_{u_{\min }}^{1} d u \int_{0}^{T} d E P(E, u) \theta(\Gamma t-1) \theta(\Gamma-v) \frac{v^{2} t}{\Gamma} .
\end{aligned}
$$

For the echo decay this would give

$$
\begin{aligned}
-\left\langle\ln \left|F_{E}(t)\right|\right\rangle_{D} & \approx \int d \Omega \int_{R_{0}}^{\infty} r^{D-1} d r \int_{u_{\min }}^{1} d u \int_{0}^{T} d E P(E, u) \theta(1-v t) \theta(1-\Gamma t) \frac{v^{2} \Gamma t^{3}}{6} \\
& +\int d \Omega \int_{R_{0}}^{\infty} r^{D-1} d r \int_{u_{\min }}^{1} d u \int_{0}^{T} d E P(E, u) \theta(v t-1) \theta(v-\Gamma) \Gamma t \\
& +\int d \Omega \int_{R_{0}}^{\infty} r^{D-1} d r \int_{u_{\min }}^{1} d u \int_{0}^{T} d E P(E, u) \theta(\Gamma t-1) \theta(\Gamma-v) \frac{v^{2} t}{\Gamma}
\end{aligned}
$$

In the next section we use these expressions to study the average Ramsey and echo decay signals that arise from the coupling of the probed TLS to thermal $\tau$-TLSs. We show that for the Ramsey dephasing there is no selfaveraging, i.e. the average result differs markedly from the typical one. For the echo dephasing, on other hand, the result is self-averaging and the dephasing rate calculated from Eq. (19) coincides with the typical case.

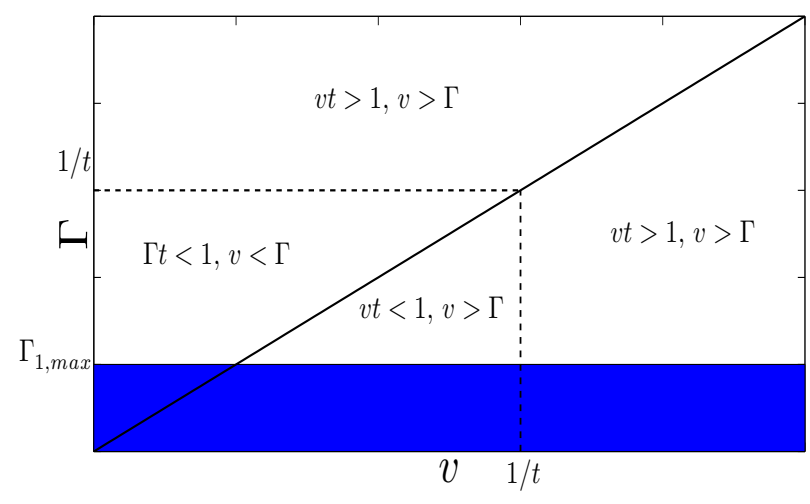

FIG. 1. (Color online) The different domains in the $v$ - $\Gamma$ plane corresponding to the various limits of a Ramsey and echo decay signals due to a single fluctuator, as described in Table I] The general expressions (18) and (19) for the averaged Ramsey and echo decay signals over an ensemble of TLSs are obtained by dividing the integrations into the various domains and setting the appropriate limits for $f_{R / E}$ in each domain. The shaded blue region shows the relevant domain for $\tau$-TLSs, $\Gamma<\Gamma_{1, \max } \ll 1 / t$.

\section{DEPHASING BY TLSS OF THE STANDARD TUNNELING MODEL ( $\tau$-TLSS)}

\section{A. Characteristics of $\tau$-TLSs}

According to Table \, the effect of an environment of thermal TLSs on the coherence properties of the probed TLS depends on the typical couplings and relaxation (switching) rates of the thermal TLSs. To estimate the relaxation rates of $\tau$-TLSs, we assume that the random transitions of each TLS are mainly due to its coupling to phonons. We will thus neglect interactions between the TLSs comprising the noisy environment. Additional relaxation mechanisms possible in superconducting circuits, such as relaxation by quasiparticle excitations, are negligible at $T<100 \mathrm{mK} \stackrel{33}{3}$ Therefore, the rate of random transitions of a single TLS is the relaxation rate due to one-phonon processes,$\stackrel{5,27}{,}$

$$
\Gamma_{1}=\frac{(2 \pi)^{3} E \Delta_{0}^{2} \gamma^{2}}{\rho h^{4}}\left(\frac{1}{v_{l}^{5}}+\frac{2}{v_{t}^{5}}\right) \operatorname{coth}(E / 2 T),
$$

where $v_{l}$ and $v_{t}$ are the sound velocities of the longitudinal and transverse modes, respectively. For a given energy splitting $E$, the maximum relaxation rate is obtained for $\Delta_{0}=E$. Moreover, for thermal TLSs with $E \approx T$, Eq. (20) yields the maximum relaxation rate

$$
\Gamma_{1, \max } \approx 2 \frac{(2 \pi)^{3} T^{3} \gamma^{2}}{\rho h^{4}}\left(\frac{1}{v_{l}^{5}}+\frac{2}{v_{t}^{5}}\right) .
$$

The relaxation rate (20) for thermal TLSs $(E<T)$ can be written in terms of $E$ and $u$ as

$$
\Gamma_{1}(E, u) \approx \Gamma_{1, \max } u\left(\frac{E}{T}\right)^{2}
$$


where we replaced $\operatorname{coth}(E / 2 T)$ in Eq. (20) by $2 T / E$ and used Eq. (21). To estimate the maximum relaxation rate

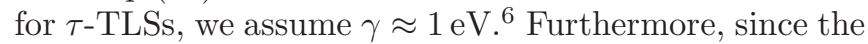
dielectric layer thickness is much smaller than the relevant phonon wavelength, the sound velocity in Eqs. (20) and (21) is set by the aluminum layers of the electrodes $\frac{14}{14}$ At $T=35 \mathrm{mK}$ (the temperature corresponding to the measurements of Ref. 18) we obtain

$$
\Gamma_{1, \max } \approx 10 \mathrm{~ms}^{-1},
$$

or equivalently, $T_{1, \min } \approx 0.1 \mathrm{~ms}$.

We next estimate the typical coupling between the probed TLS and its nearest thermal TLS. The typical distance between the probed TLS and its nearest thermal $\tau$-TLS, $R_{T}$, can be estimated using the relations

$$
\begin{aligned}
& R_{T, 3 D}^{3} \int_{u_{\min }}^{1} d u \int_{0}^{T} d E P(E, u)=1 \quad(3 \mathrm{D}), \\
& R_{T, 2 D}^{2} d \int_{u_{\min }}^{1} d u \int_{0}^{T} d E P(E, u)=1 \quad(2 \mathrm{D}),
\end{aligned}
$$

where $u_{\min }$ is a lower cutoff for the parameter $u$ and $d$ is the tunnel barrier thickness $(d \approx 3 \mathrm{~nm})$. Here we provide the estimates in three and two dimensions (3D and 2D, respectively) due to the quasi-2D structure of the amorphous tunnel barrier. In the framework of the STM, one usually assumes a constant DOS, namely $P_{0}$ in Eq. (17) is a constant. The central dimensionless parameter of the STM is the tunneling strength $C_{0}=P_{0} \gamma^{2} / \rho v^{2}=P_{0} R_{0}^{3} J_{0} \approx 10^{-3} \stackrel{3}{\underline{3}}$ where $R_{0}$ and $J_{0}$ are the typical distance and typical interaction between nearest neighbors $\tau$-TLSs, respectively.

Substituting Eq. (17) into Eq. (24), we readily obtain $R_{T, 3 D}^{3}=R_{T, 2 D}^{2} d \approx 1 /\left(P_{0} \xi T\right)$ with $\xi=\ln \left(1 / u_{\min }\right)$. Inserting these distances in the interaction coefficients [Eqs. (3) and (4)], we find the typical coupling strength between the probed TLS and its nearest thermal $\tau$-TLS,

$$
\begin{aligned}
& J_{T}=C_{0} \xi T \sim 2 \pi \cdot 10 \mathrm{MHz} \quad(3 \mathrm{D}), \\
& J_{T}=C_{0} \xi T\left(\frac{d}{R_{T, 3 D}}\right)^{3 / 2} \sim 2 \pi \cdot 1 \mathrm{MHz} \quad(2 \mathrm{D}),
\end{aligned}
$$

where we assumed the usual values $C_{0} \approx 10^{-3}$ and $\xi \approx 20$. For the $2 \mathrm{D}$ estimate we also used $R_{0} \approx d / 3$ and $J_{0} \approx 1 \mathrm{~K} \approx 2 \pi \cdot 20 \mathrm{GHz}$. As shown in Ref. 18 , the $2 \mathrm{D}$ estimate of $J_{T}$ is in good agreement with the observed magnitude of the Ramsey dephasing rate (see more details below), whereas the 3D estimate yields somewhat larger rates than observed experimentally. This agrees with the quasi-2D structure of the junction barrier and suggests that TLSs reside only in the amorphous tunnel barrier.

Comparison of Eqs. (23) and (25) reveals that thermal $\tau$-TLSs satisfy $J_{T} \gg \Gamma_{1, \max }$. We recall Eq. (11) and take into account that typically, $\cos \theta_{j}=O(1)$. Therefore, in a typical sample the most significant thermal $\tau$-TLSs (i.e. those located at $r \sim R_{T}$ ) are characterized by $v \gg \Gamma$, except in the very close vicinity of the symmetry point of the probed TLS, $\Delta_{p}=0$ (hence $\cos \theta_{p}=0$ ), which can not be distinguished within the resolution of $\Delta_{p}$ in the experiment. Therefore, in the case of $\tau$-TLSs we should study the $\Delta_{p}$-dependence of the dephasing rates assuming the presence of fluctuators which are slow and strongly coupled to the probed TLS, as sketched schematically in Fig. 2

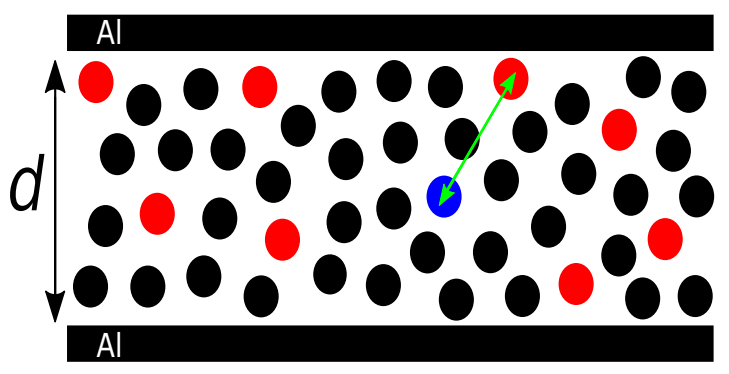

FIG. 2. (Color online) Schematic view of TLSs (circles) in the aluminum oxide tunnel barrier (of width $d$ ) of a Josephson junction. The probed TLS is shown in blue and non-thermal TLSs are shown in black. Thermal TLSs are denoted in red and the typical distance $R_{T}$ between the probed TLS and its nearest thermal TLS is shown by a green arrow. Thermal TLSs are typically in the regime $v \gg \Gamma$. As discussed in the text, Ramsey dephasing is dominated by few thermal TLSs located at $r \sim R_{T}$ whereas echo dephasing is caused by a large number of thermal TLSs.

\section{B. Ramsey dephasing rate}

As explained above, the contribution of $\tau$-TLSs to the Ramsey and echo decay signals should be treated within the limit $v \gg \Gamma$. Under these circumstances the third line of Eqs. (18) and (19) can be disregarded. Furthermore, we examine now the regime of purely static dephasing, $\Gamma_{1, \max } t \ll 1$, which is the relevant regime for the Ramsey dephasing studied in Ref. 18. Since the observed dephasing times $T_{\varphi, R}=\Gamma_{\varphi, R}^{-1}$ are on the order of microseconds, the condition $\Gamma_{1, \max } t \ll 1$ indeed holds at the relevant times $t<T_{\varphi, R}$.

In this case the average Ramsey decay signal due to $\tau$-TLSs can be estimated as

$$
\begin{aligned}
& \left\langle\ln \left|F_{R}(t)\right|\right\rangle_{D} \\
& =\int d \Omega \int_{R_{0}}^{\infty} r^{D-1} d r \int_{u_{\min }}^{1} d u \int_{0}^{T} d E P(E, u) \ln |\cos (v t)|,
\end{aligned}
$$

where $v(r, u)=J_{0}\left(R_{0} / r\right)^{3} \sqrt{1-u} \cos \theta_{p}$ [see Eqs. (3), (4), (11)]. Using Eq. (17) and introducing an auxiliary vari- 
able $x \equiv t J_{0}\left(R_{0} / r\right)^{3}\left|\cos \theta_{p}\right|$ we obtain for $D=3$

$$
\begin{aligned}
& \left\langle\ln \left|F_{R}(t)\right|\right\rangle_{D}=\frac{2 \pi}{3} C_{0} T t\left|\cos \theta_{p}\right| \\
& \times \int_{0}^{x_{t}} \frac{d x}{x^{2}} \int_{u_{\min }}^{1} d u \frac{\ln |\cos (x \sqrt{1-u})|}{u \sqrt{1-u}},
\end{aligned}
$$

where $x_{t} \equiv t J_{0}\left|\cos \theta_{p}\right|$. Setting $J_{0} \approx 1 \mathrm{~K} \approx 2 \pi \cdot 20 \mathrm{GHz}$, we observe that at all relevant times (of the order of the dephasing time $\left.\Gamma_{\varphi, R}^{-1}\right) x_{t} \gg 1$, except in a very small vicinity of the symmetry point $\cos \theta_{p}=0$. Thus we take the limit $x_{t} \rightarrow \infty$ in Eq. (27). The integral over $x$ converges, whereas integration over $u$ gives a result of the order of $\xi=\ln \left(1 / u_{\min }\right)$. Thus we obtain (for $D=3$ )

$$
\left\langle\ln \left|F_{R}(t)\right|\right\rangle_{D} \sim-J_{T}\left|\cos \theta_{p}\right| t .
$$

A similar calculation for $D=2$ gives

$$
\left\langle\ln \left|F_{R}(t)\right|\right\rangle_{D} \sim-\left[J_{T}\left|\cos \theta_{p}\right|\right]^{2 / 3} t^{2 / 3} .
$$

The coupling strength between the probed TLS and its closest thermal TLS, $J_{T}$, is defined in Eqs. (25) for both $D=3$ and $D=2$. The results (28) and (29) turn out to be non-self-averaging.

Indeed, in a typical situation there is a closest thermal TLS with maximum coupling $v_{\max }^{T} \sim J_{T}\left|\cos \theta_{p}\right| \gg$ $\Gamma_{1, \max }$. At very short times, $t \ll 1 / v_{\max }^{T}$, one obtains $\ln \left|F_{R}(t)\right|=-(1 / 2) t^{2} \sum_{j} v_{j}^{2}$. Since $v_{j} \sim 1 / r_{j}^{3}$ the sum is dominated by the few closest thermal TLSs and in both $D=3$ and $D=2$

$$
\ln \left|F_{R}^{t y p}(t)\right| \sim-\left[J_{T} \cos \theta_{p}\right]^{2} t^{2} .
$$

Both the average [Eqs. (28) and (29)] and the typical [Eq. 30)] results give the dephasing rate ${ }^{34}$

$$
\Gamma_{\varphi, R} \approx J_{T}\left|\cos \theta_{p}\right|
$$

yet with a very different functional time dependence. At short times, $\Gamma_{\varphi, R} t \ll 1$, the typical result gives a much weaker Ramsey decay than would be naively expected from the average result. At longer times, $\Gamma_{\varphi, R} t \gg 1$, the difference between the average and the typical results is even more striking. The typical decay function $F_{R}^{t y p}(t)$ at such times oscillates between positive and negative values, $, 25,26$ which means that the envelope of Ramsey fringes has points of zero amplitude where phase slips occur. In contrast, the average result is monotonically decaying. This situation is demonstrated in Fig. 3 .

As demonstrated experimentally in Refs. 17 and 18, the energy bias of the probed TLS changes linearly with an external strain (which, in turn, is proportional to a piezo voltage $V$ ). Therefore, close to the symmetry point $\Delta_{p}=0$, we can write $\cos \theta_{p}=\Delta_{p} / E_{p}$ as

$$
\cos \theta_{p}=\frac{\Delta_{p}}{E_{p}}=\frac{\Delta_{p}}{\sqrt{\Delta_{p}^{2}+\Delta_{0, p}^{2}}} \approx \frac{\Delta_{p}}{\Delta_{0, p}} .
$$

Combining Eqs. (31) and (32), one finds $\Gamma_{\varphi, R} \propto\left|\Delta_{p}\right|$, i.e. the Ramsey dephasing rate is expected to change linearly

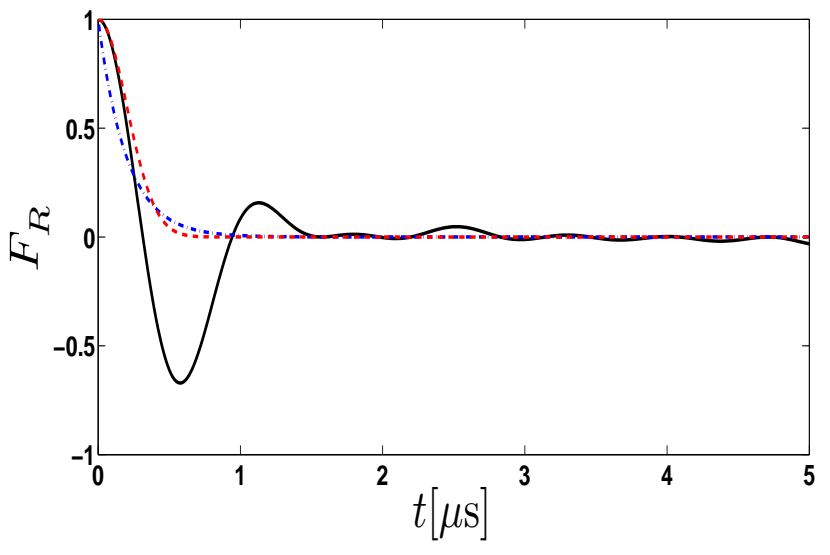

FIG. 3. (Color online) Comparison of $e^{\left\langle\ln \left|F_{R}(t)\right|\right\rangle_{D}}$ obtained by averaging over an ensemble of slow fluctuators with $\Gamma \ll$ $\Gamma_{\varphi, R}$ as given by Eq. (28) (dashed-dotted blue line), and of $F_{R}(t)$ for a specific realization of six thermal fluctuators with couplings $v=2 \pi \times\{5,1,0.8,0.5,0.4,0.2\} \mathrm{MHz}$ and relaxation rates $\Gamma=\{5,0.5,8,0.8,0.1,3\} \mathrm{ms}^{-1}$ (solid black line). At short times, $t \ll 1 / v_{\max }^{T} \sim 0.1 \mu \mathrm{s}$, the Ramsey decay is well approximated by a typical Gaussian decay as given in Eq. (30) (dashed red line).

with $\Delta_{p}$. However, this linear dependence is expected to hold as long as the Ramsey dephasing is dominated by the same decohering thermal TLSs. As the strain is varied the asymmetry bias $\Delta_{j}$ of the decohering TLSs will change as well, similarly to the probed TLS. As a result, at some point the energy of a decohering TLS will exceed the thermal energy. At the same time, other TLSs which were non-thermal at lower strains will become thermal. Assuming that thermal TLSs are coupled to the strain similarly as the probed TLS, one expects that once $\Delta_{p}$ is changed by $T \sim 2 \pi \cdot 1 \mathrm{GHz}$, the dominant decohering TLSs will be different ones. This gives rise to a nonregular behavior, reflected in a change of slope or small irregularities in the Ramsey dephasing rate as a function of strain. Such features are indeed observed in the $\Delta_{p}$-dependence of the Ramsey dephasing rate $\frac{18}{}$ and are strongly suggestive of the predominance of single thermal TLSs in the Ramsey dephasing. As a result, we expect the pure linear behavior to be relevant at a window $\left|\Delta_{p}\right|<2 \pi \cdot 1 \mathrm{GHz}$ around the symmetry point of the probed TLS. Indeed, for the four TLSs studied in Ref. 18, the estimate for $J_{T}$ in $2 \mathrm{D}$ is in excellent agreement with the fitted slope of the linear line in the region $\left|\Delta_{p}\right|<2 \pi \cdot 1 \mathrm{GHz}$.

Equations 25) and (31) also allow us to predict the temperature dependence (for low temperatures, $T<$ $100 \mathrm{mK}$ ) of the Ramsey dephasing rate. At a fixed strain, these equations predict $\Gamma_{\varphi, R} \propto T$ for the $3 \mathrm{D}$ case and $\Gamma_{\varphi, R} \propto T^{3 / 2}$ for the $2 \mathrm{D}$ case. For an energydependent DOS of the form $n_{\tau}(E) \propto E^{\eta}, \frac{35-37}{3}$ the corresponding temperature dependence is $\Gamma_{\varphi, R} \propto T^{1+\eta}$ and $\Gamma_{\varphi, R} \propto T^{3(1+\eta) / 2}$, for $3 \mathrm{D}$ and $2 \mathrm{D}$, respectively. 


\section{Echo dephasing rate}

To estimate the contribution of $\tau$-TLSs to the ensemble average of the echo decay signal, we have to calculate the first two integrals of Eq. (19). For simplicity, we perform the integration in $3 \mathrm{D}$. The results in $2 \mathrm{D}$ are qualitatively similar and given in appendix $\mathrm{A}$. The contribution to the first integral comes from TLSs for which $v t<1$. Since for $\tau$-TLSs $v \gg \Gamma$, the condition $\Gamma t<1$ is automatically satisfied. Hence, the contribution to this integral comes from TLSs which are located at distances $r>R_{*}$ from the probed TLS, where $R_{*}^{3}=2 J_{0} R_{0}^{3} \sqrt{1-u}\left|\cos \theta_{p}\right| t$. Therefore,

$$
\begin{aligned}
& \int_{R_{0}}^{\infty} 4 \pi r^{2} d r \int_{0}^{1} d u \int_{0}^{T} d E P(E, u) \theta(1-v t) \theta(1-\Gamma t) \frac{v^{2} \Gamma t^{3}}{6} \\
& \approx \frac{2}{3} t^{3} \cos ^{2} \theta_{p} P_{0}\left(J_{0} R_{0}^{3}\right)^{2} \Gamma_{1, \max } \int_{R_{*}}^{\infty} \frac{4 \pi}{r^{4}} d r \int_{0}^{1} \frac{\sqrt{1-u}}{2} d u \int_{0}^{T}\left(\frac{E}{T}\right)^{2} d E \\
& =\frac{2 \pi}{27} t^{2}\left|\cos \theta_{p}\right| P_{0} R_{0}^{3} J_{0} T \Gamma_{1, \max } \approx \frac{2 \pi}{27} t^{2} J_{T}\left|\cos \theta_{p}\right| \Gamma_{1, \max } \xi^{-1}
\end{aligned}
$$

where $J_{T}$ is given by the first of Eqs. (25). The main contribution to the second integral comes from the close
TLSs, for which $r<R_{*}$. Again, the condition $v \gg \Gamma$ for $\tau$-TLSs ensures that the second step function is always unity. Thus,

$$
\begin{aligned}
& \int_{R_{0}}^{\infty} 4 \pi r^{2} d r \int_{0}^{1} d u \int_{0}^{T} d E P(E, u) \theta(v t-1) \theta(v-\Gamma) \Gamma t \\
& \approx t P_{0} \Gamma_{1, \max } \int_{0}^{R_{*}} 4 \pi r^{2} d r \int_{0}^{1} \frac{d u}{2 \sqrt{1-u}} \int_{0}^{T}\left(\frac{E}{T}\right)^{2} d E \\
& =\frac{4 \pi}{9} t^{2}\left|\cos \theta_{p}\right| P_{0} R_{0}^{3} J_{0} T \Gamma_{1, \max } \approx \frac{4 \pi}{9} t^{2} J_{T}\left|\cos \theta_{p}\right| \Gamma_{1, \max } \xi^{-1}
\end{aligned}
$$

Neglecting a numerical prefactor of order unity, the sum of Eqs. (33) and (34) gives

$$
\left\langle\ln \left|F_{E}(t)\right|\right\rangle_{D} \approx-t^{2} J_{T}\left|\cos \theta_{p}\right| \Gamma_{1, \max } \xi^{-1} .
$$

Let us check if this result is self-averaging. To this end, we estimate the number of thermal TLSs, $N_{*}$, in a sphere of radius $R_{*}$ at time $t \approx \Gamma_{\varphi, E}^{-1}$, with $\Gamma_{\varphi, E}^{-1}$ the echo dephasing rate

$$
\Gamma_{\varphi, E} \approx \sqrt{\Gamma_{1, \max } \xi^{-1} J_{T}\left|\cos \theta_{p}\right|}
$$

This yields

$$
\begin{aligned}
N_{*} & =\frac{4 \pi}{3} \int_{u_{\min }}^{1} d u \int_{0}^{T} d E P(E, u) R_{*}^{2}\left(t=\Gamma_{\varphi, E}^{-1}\right) \\
& \approx \frac{4 \pi}{3} J_{T}\left|\cos \theta_{p}\right| \Gamma_{\varphi, E}^{-1} \approx \frac{4 \pi}{3} \sqrt{\frac{\xi J_{T}}{\Gamma_{1, \max }}\left|\cos \theta_{p}\right|} \\
& \approx 1500 \sqrt{\left|\cos \theta_{p}\right|} .
\end{aligned}
$$

Therefore, except for strains very close to the symmetry point (which are outside the resolution of the experiment), a sphere of radius $R_{*}$ contains many thermal TLSs. The contribution of $\tau$-TLSs to the echo decay signal is thus self-averaging, and the echo dephasing rate is given by Eq. (36). Since thermal $\tau$-TLSs fluctuate very slowly, we expect the echo dephasing rate to be much smaller than the Ramsey dephasing rate. Using Eqs. (31) and (36), we find

$$
\frac{\Gamma_{\varphi, R}}{\Gamma_{\varphi, E}}=\sqrt{\frac{\xi J_{T}}{\Gamma_{1, \max }}\left|\cos \theta_{p}\right|} \approx 400 \sqrt{\left|\cos \theta_{p}\right|} .
$$

Thus, the echo protocol is very efficient in suppressing the noise caused by fluctuations of thermal $\tau$-TLSs. This contradicts the experimental observation, which shows that the echo dephasing rate is much larger than the contribution of $\tau$-TLSs, Eq. (36). Furthermore, the $\left|\Delta_{p}\right|^{1 / 2}$ dependence of the echo dephasing rate on the asymmetry bias, predicted by Eq. (36), is also in disagreement with the experimental result, which shows a quadratic dependence. By repeating the calculations in 2D one finds 
$\Gamma_{\varphi, E} \propto\left|\Delta_{p}\right|^{2 / 5}$ (see appendix @), which also contradicts the experiment. In fact, among the three integrals of Eq. (19) only the third one can explain the quadratic dependence of the echo dephasing rate. The contribution to this integral comes from fluctuators with $\Gamma t>1$ and $\Gamma>v$, namely from fast fluctuators that are weakly coupled to the probed TLS. Such fluctuators are in the opposite regime of that corresponding to $\tau$-TLSs. In the next section we show that $S$-TLSs, proposed in Ref. 23, obey the above conditions and form a possible source of such a noise. We estimate the order of magnitude of the echo dephasing rate due to such TLSs and examine their relevance to the results of Ref. 18 .

\section{DECOHERENCE BY TLSS STRONGLY INTERACTING WITH STRAIN}

The STM assumes that the coupling of TLSs to the strain is unique or taken from a narrow distribution. Here we consider the possibility that TLSs with much stronger coupling to the strain generically exist in amorphous solids, as has recently been proposed in Ref. 23 . We show that the existence of a few or even a single thermal and strongly coupled to strain TLSs within the amorphous barrier produces echo dephasing rate which is in agreement with both the magnitude and the strain dependence found experimentally.

\section{A. The model with two types of TLSs}

The theory presented in Ref. 23 suggests the existence of two types of TLSs, characterized by different coupling strengths to strain fields. Weakly and strongly coupled TLSs are characterized by coupling strength $\gamma_{\tau}$ and $\gamma_{S}$ to strain fields, respectively. The small dimensionless parameter of the theory is then $g \equiv \gamma_{\tau} / \gamma_{S}$, which is expected 23.38 .39 to lie in the range $g \approx 0.01-0.03$. The bimodality of the coupling strengths of TLSs to strain fields also modifies the phonon-mediated interactions between the TLSs $\stackrel{\underline{7}-\underline{9}}{-}$ The Hamiltonian (2) is then generalized to $\underline{23,40}$

$$
\hat{\mathcal{H}}_{\tau S}=\frac{1}{2} \sum_{i \neq j}\left(J_{i j}^{\tau \tau} \hat{\tau}_{z, i} \hat{\tau}_{z, j}+J_{i j}^{\tau S} \hat{\tau}_{z, i} \hat{S}_{z, j}+J_{i j}^{S S} \hat{S}_{z, i} \hat{S}_{z, j}\right),
$$

where $\hat{\tau}_{z, i}$ and $\hat{S}_{z, j}$ are the Pauli matrices that represent the $\tau$ - and $S$-TLSs at sites $i$ and $j$, and the interaction coefficients are similar to those of Eqs. (3) and (4),

$$
J_{i j}^{a b}=\frac{c_{i j}^{a b} \gamma_{a} \gamma_{b}}{\rho v^{2} R_{i j}^{3}}=c_{i j}^{a b} J_{0}^{a b}\left(\frac{R_{0}}{R_{i j}}\right)^{3} \quad a, b=\tau, S .
$$

with $J_{0}^{a b}=\gamma_{a} \gamma_{b} /\left(\rho v^{2} R_{0}^{3}\right)$ and $R_{0}$ being the distance between nearest neighbor TLSs.
The implications of the interactions (39) between the two types of TLSs on their single particle density of states (DOS) have been studied in detail in Refs. 41 and 42 . It was found that a pseudo-gap exists in the DOS of $S$-TLSs at low energies $E<J_{0}^{\tau S}=g J_{0}^{S S} \sim 10 \mathrm{~K} \sim 2 \pi \cdot 200 \mathrm{GHz}$. In contrast, the $\tau$-TLS DOS is well described by a Gaussian with width $\sim J_{0}^{\tau S}$. Thus, at low energies $E \ll J_{0}^{\tau S} \sim$ $10 \mathrm{~K}, S$-TLSs are much more scarce than $\tau$-TLSs. As a consequence, most low-temperature properties, such as specific heat and thermal conductivity, are dominated by the weakly interacting $\tau$-TLSs, which can be identified as the well-known TLSs of the STM.

\section{B. Characteristics of $S$-TLSs}

We estimate the maximum relaxation rate of thermal $S$-TLSs and their typical coupling to the probed $\tau$-TLS by repeating the calculations of Sec. IIIA The relaxation rate is given by Eqs. (20)-(22), with $\gamma_{\tau}$ replaced by $\gamma_{S}=\gamma_{\tau} / g$. This yields the maximum relaxation rate

$$
\Gamma_{1, \max }^{(S)}=\frac{\Gamma_{1, \max }^{(\tau)}}{g^{2}} \approx 10-100 \mu \mathrm{s}^{-1},
$$

where we used Eq. (23) and $g \approx 0.01-0.03$.

In appendix B we derive the typical distance between the probed TLS and its nearest thermal $S$-TLS and conclude that it is somewhat larger than the size of the amorphous tunnel barrier used in Ref. $18(\sim 100 \mathrm{~nm})$. We therefore assume the existence of one or few $S$-TLSs in the amorphous layer, relying also on the possibility of an enhanced $S$-TLSs DOS on the surface $\underline{\underline{43}}$ Thus, the typical distance between the probed TLS and its nearest thermal $S$-TLS is of the order of the amorphous layer size which gives a typical interaction

$$
J_{T}^{\tau S} \approx 2 \pi \times(1-10) \mathrm{MHz} .
$$

Therefore, one finds that for $S$-TLSs $J_{T}^{\tau S}<\Gamma_{1, \max }^{(S)}$. It thus follows that in a typical sample the thermal $S$-TLSs are characterized by $v<\Gamma$, as shown schematically in Fig. 4 (cf. Fig. 2). As argued above, such fluctuators give rise to a quadratic dependence of the echo dephasing rate on the asymmetry bias $\Delta_{p}$. We now study in more detail the effects of $S$-TLSs on the Ramsey and echo decay signals.

\section{Ramsey and Echo dephasing rates due to $S$-TLSs}

For $S$-TLSs, which satisfy $v<\Gamma$, we neglect the second integral of Eqs. (18) and (19). At short times, $t<1 / \Gamma_{\max }^{(S)}$, only the first integral of Eqs. (18) and (19) contributes. Similarly to Eq. (30), the typical Ramsey decay signal is governed by the nearest thermal $S$-TLSs, 


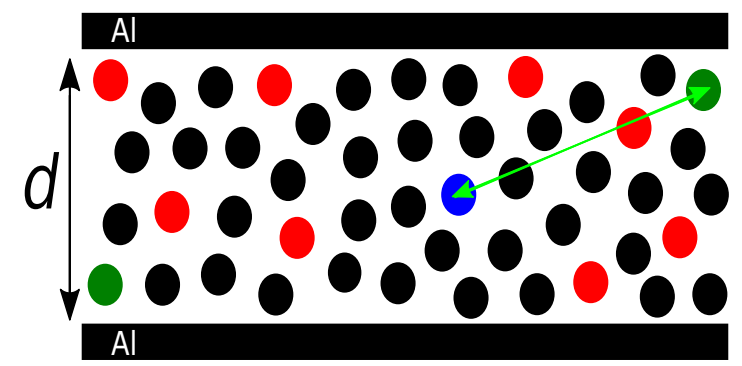

FIG. 4. (Color online) Schematic view of a bath of thermal $S$-TLSs (green circles) and $\tau$-TLSs (red circles) coupled to the probed TLS (blue circle). Non-thermal TLSs are shown in black circles. Thermal $S$-TLSs are much more scarce than thermal $\tau$-TLSs and thus located much farther from the probed TLS (the typical distance $R_{T}$ is shown by a green arrow). Thus, in contrast to $\tau$-TLSs, $S$-TLSs are fast and weakly coupled to the probed TLS, i.e. they are typically in the regime $\Gamma t>1$ and $v<\Gamma$.

i.e.

$$
\ln F_{R}^{t y p}(t) \approx-\left[J_{T}^{\tau S} \cos \theta_{p}\right]^{2} t^{2}
$$

This quantity is very small for $t<1 / \Gamma_{1, \max }^{(S)}$, so dephasing has not yet occurred at such short times. The typical echo decay signal is of the order

$$
\ln F_{E}^{t y p}(t) \approx-\left[J_{T}^{\tau S} \cos \theta_{p}\right]^{2} \Gamma_{1, \max }^{(S)} t^{3}
$$

which is also very small. It should also be emphasized that in contrast to $\tau$-TLSs, the contribution of $S$-TLSs to the average echo decay signal is not self-averaging, since the first integral of Eq. (19) is dominated by rare samples in which the nearest thermal $S$-TLS is located at $r \sim R_{0}$.

As $t$ becomes longer than $1 / \Gamma_{1 \text { max }}^{(S)}$, both Ramsey and echo decay signals are dominated by the third integral of Eqs. (18) and (19). Since this integral also involves $v^{2}$, the main contribution comes from the thermal $S$-TLS for which the quantity $v^{2} / \Gamma$ is maximum. Thus, in a typical sample we expect the order of magnitude of the contribution of $S$-TLSs to the Ramsey and echo decay signals to be

$$
\ln F_{R}^{t y p}(t)=\ln F_{E}^{t y p}(t) \approx-\frac{\left[J_{T}^{\tau S} \cos \theta_{p}\right]^{2}}{\Gamma_{1, \max }^{(S)}} t .
$$

The Ramsey and echo dephasing rates in $2 \mathrm{D}$ are thus

$$
\begin{aligned}
\Gamma_{\varphi, R} & =\Gamma_{\varphi, E} \approx \frac{\left(J_{T}^{\tau S}\right)^{2}}{\Gamma_{1, \max }^{(S)} \cos ^{2} \theta_{p}} \\
& \approx 2 \pi \times \cos ^{2} \theta_{p} \mathrm{MHz}
\end{aligned}
$$

where in the last step we used Eqs. (41) and (42). Close to the symmetry point Eq. (46) predicts a quadratic dependence of the echo dephasing rate on the asymmetry bias, in agreement with the experiment. Moreover, the order of magnitude of the echo dephasing rate is also in line with the experimental result. We note that the above result holds provided that at least one $S$-TLS exists within the amorphous layer. Otherwise, we expect the echo dephasing to be negligible. This is indeed observed in one out of four TLSs in the experiment in Ref. 18.

The result (46) for the Ramsey and echo dephasing rates due to fast fluctuators can also be understood in terms of the Gaussian approximation, since in the regime $\Gamma>v$ the results of the spin-fluctutator model are expected to coincide with the Gaussian approximation ${ }^{26}$ Within the Gaussian approximation, where $X(t)$ in Eqs. (10), (12) and (13) is assumed to have a Gaussian statistics, one ends up with

$$
\begin{aligned}
& -\ln F_{R / E}^{G}(t)=\frac{t^{2}}{2} \int_{-\infty}^{\infty} \frac{d \omega}{2 \pi} S_{X}(\omega) K_{R / E}(\omega, t), \\
& K_{R}(\omega, t)=\operatorname{sinc}^{2}\left(\frac{\omega t}{2}\right), \\
& K_{E}(\omega, t)=\sin ^{2}\left(\frac{\omega t}{4}\right) \operatorname{sinc}^{2}\left(\frac{\omega t}{4}\right),
\end{aligned}
$$

where $\operatorname{sinc}(x) \equiv \sin (x) / x$ and $S_{X}(\omega)$ is the noise spectral density, i.e. the Fourier transform of the correlation function $S_{X}(t)=\langle X(t) X(0)\rangle$. If $S_{X}(\omega)$ is approximately constant for $|\omega| \lesssim \Gamma_{\varphi, E / R}, \frac{44}{}$ namely $X(t)$ is a white noise, then $-\ln F_{R / E}^{G}(t)=\Gamma_{\varphi, R / E} t$ where

$$
\Gamma_{\varphi, R}=\Gamma_{\varphi, E} \approx \frac{1}{2} S_{X}(\omega=0) .
$$

Using Eq. (10) and assuming independent fluctuators, one finds 26

$$
S_{X}(\omega)=\sum_{j} v_{j}^{2} S_{j}(\omega)=\cos ^{2} \theta_{p} \sum_{j} J_{j}^{2} \cos ^{2} \theta_{j} S_{j}(\omega),
$$

where $S_{j}(\omega)$ is the noise spectral density of a single fluctuator,

$$
S_{j}(\omega)=\frac{2 \Gamma_{j}}{\Gamma_{j}^{2}+\omega^{2}} .
$$

Equation (48) then yields

$$
\Gamma_{\varphi, R}=\Gamma_{\varphi, E} \approx \sum_{j} \frac{J_{j}^{2} \cos ^{2} \theta_{j}}{\Gamma_{j}} \cos ^{2} \theta_{p}
$$

For an interaction which falls off as $1 / R^{3}$, the sum is dominated by a few closet fluctuators and the result (51) is of the same order of magnitude as (46).

\section{DISCUSSION AND CONCLUSIONS}

We have investigated the strain-dependent dephasing rates of individual TLSs in the amorphous tunnel barrier 
of a superconducting phase qubit, as recently studied in Ref. 18. The interaction between the probed TLS and thermal standard $(\tau-)$ TLSs described in the framework of the STM is capable of explaining the observed Ramsey dephasing rate. This includes:

(1) The linear dependence on the applied strain and hence on the bias energy of the probed TLS, $\Gamma_{\varphi, R} \propto$ $\left|\Delta_{p}\right|$, near the symmetry point, $\Delta_{p}=0$. It is a consequence of the small relaxation rate of thermal $\tau$-TLSs compared to their interaction with the probed TLS.

(2) The deviation from linear behavior and the appearance of irregularities when $\left|\Delta_{p}\right|$ is varied by an amount of the order of the thermal energy $T \approx$ $2 \pi \cdot 1 \mathrm{GHz}$. Such features are expected since the theory predicts the Ramsey dephasing to be dominantly caused by a small number of TLSs. As the strain is varied, some of these TLSs are no longer thermal while other TLSs will become so and contribute to the Ramsey dephasing.

(3) The magnitude of the Ramsey dephasing rate. Using standard estimates for the coupling of $\tau$-TLSs to strain fields, $\gamma \approx 1 \mathrm{eV}$, and for the dimensionless "tunneling strength" $C_{0}=P_{0} \gamma^{2} / \rho v^{2} \approx 10^{-3}$, it is shown that the order of magnitude of the Ramsey dephasing rate is in excellent agreement with the experimental result. The estimate for a quasi-2D configuration is in better agreement than that in a $3 \mathrm{D}$ configuration which is consistent with the experimental setup. Although we considered elastic interactions between $\tau$-TLSs, it should be noted that our analysis and results can be carried through to the case where electric dipole interactions are present between $\tau$-TLSs. For typical values of the dipole moment, of the order of 5 Debye, $12,45-47$ electric dipole interactions are expected to be of the same order of magnitude as elastic interactions between $\tau$-TLSs and the results are the same.

Furthermore, we predict the dependence of the Ramsey dephasing rate on temperature for both $2 \mathrm{D}$ and $3 \mathrm{D}$ geometries for an arbitrary power-law energy dependence of the TLS DOS at low energies.

For the echo dephasing, our analysis shows that the contribution of $\tau$-TLSs is very much reduced $\left(\Gamma_{\varphi, E} / \Gamma_{\varphi, R} \sim 0.01\right.$ for the typical strains studied in Ref. 18) and the bias-dependence is predicted to be $\Gamma_{\varphi, E} \propto\left|\Delta_{p}\right|^{0.4}\left(\right.$ or $\Gamma_{\varphi, E} \propto\left|\Delta_{p}\right|^{0.5}$ in 3D). Such small dephasing rates are observed only in one out of four TLSs studied in Ref. 18. In the other TLSs the echo dephasing rate is higher and varies quadratically with the applied strain, implying the presence of energy fluctuations on a time scale $\lesssim 1 \mu \mathrm{s}$. We suggest that TLSs that are strongly coupled to strain 23 and therefore fast fluctuating may be the dominant source for noise and governing echo dephasing at low temperatures. The properties of such TLSs, in particular their scarcity compared to the standard TLSs of the STM, result in both the order of magnitude and the strong fluctuations of the echo dephasing as seen in the experiment ${ }^{18}$.

\section{ACKNOWLEDGMENTS}

We acknowledge fruitful discussions with J. Lisenfeld. This work was supported by the German-Israeli Foundation (GIF) and by the DFG Research Grant SCHO 287/7-1, SH 81/2-1.

\section{Appendix A: Calculation of the echo dephasing rate due to $\tau$-TLSs in $2 \mathrm{D}$}

We give here the results of the calculations performed in Sec. IIIC for the 2D case. The integrals 33 and 34 in $2 \mathrm{D}$ give

$$
\begin{aligned}
& d \int_{R_{0}}^{\infty} 2 \pi r d r \int_{0}^{1} d u \int_{0}^{T} d E P(E, u) \theta(1-v t) \theta(1-\Gamma t) \frac{v^{2} \Gamma t^{3}}{6} \approx \frac{\pi d}{120 \xi R_{0}}\left(\frac{J_{T, 3 D}}{J_{0}}\right)^{1 / 3} t^{5 / 3}\left[J_{T, 3 D}\left|\cos \theta_{p}\right|\right]^{2 / 3} \Gamma_{1, \max } \\
& d \int_{R_{0}}^{\infty} 2 \pi r d r \int_{0}^{1} d u \int_{0}^{T} d E P(E, u) \theta(v t-1) \theta(v-\Gamma) \Gamma t \approx \frac{2 \pi d}{5 \xi R_{0}}\left(\frac{J_{T, 3 D}}{J_{0}}\right)^{1 / 3} t^{5 / 3}\left[J_{T, 3 D}\left|\cos \theta_{p}\right|\right]^{2 / 3} \Gamma_{1, \max }, \quad(\mathrm{A} 2)
\end{aligned}
$$

where $J_{T, 3 D}$ is given by the first of Eqs. (25). The sum of Eqs. A1 and (A2) yields [cf. Eq. 35]

$$
\begin{aligned}
\left\langle\ln \left|F_{E}(t)\right|\right\rangle_{D} & \approx-\frac{d}{\xi R_{0}}\left(\frac{J_{T, 3 D}}{J_{0}}\right)^{1 / 3} t^{5 / 3}\left[J_{T, 3 D}\left|\cos \theta_{p}\right|\right]^{2 / 3} \\
& \times \Gamma_{1, \max }
\end{aligned}
$$

where a numerical coefficient of order unity is omitted. The echo dephasing rate is thus

$$
\Gamma_{\varphi, E} \approx\left[\frac{J_{T, 3 D} \Gamma_{1, \max } d}{\xi R_{0}\left(J_{0}\right)^{1 / 3}}\right]^{3 / 5}\left|\cos \theta_{p}\right|^{2 / 5}
$$


Equation (37) takes the form

$$
\begin{aligned}
N_{*} & =\pi d \int_{u_{\min }}^{1} d u \int_{0}^{T} d E P(E, u) R_{*}^{2}\left(t=\Gamma_{\varphi, E}^{-1}\right) \\
& \approx \frac{\pi d}{R_{0}}\left(\frac{J_{T, 3 D}}{J_{0}}\right)^{1 / 3}\left[\frac{J_{T, 3 D}\left|\cos \theta_{p}\right|}{\Gamma_{\varphi, E}}\right]^{2 / 3} \\
& \approx \pi\left[\frac{d^{3 / 2} \xi J_{T, 3 D}}{R_{0}^{3 / 2} \Gamma_{1, \max }} \sqrt{\frac{J_{T, 3 D}}{J_{0}}}\left|\cos \theta_{p}\right|\right]^{2 / 5} \\
& \approx 100\left|\cos \theta_{p}\right|^{2 / 5} .
\end{aligned}
$$

Therefore, except for strains very close to the symmetry point (which are outside the resolution of the experiment), a circle of radius $R_{*}$ contains many thermal TLSs. The contribution of $\tau$-TLSs to the echo decay signal in $2 \mathrm{D}$ is thus also self-averaging, and the echo dephasing rate is given by Eq. (A4). The ratio between the echo dephasing rate and the Ramsey dephasing rate is

$$
\begin{aligned}
\frac{\Gamma_{\varphi, R}}{\Gamma_{\varphi, E}} & =\left(\frac{\xi J_{T, 3 D}\left|\cos \theta_{p}\right|}{\Gamma_{1, \max }}\right)^{3 / 5}\left(\frac{d}{R_{0}}\right)^{9 / 10}\left(\frac{J_{T, 3 D}}{J_{0}}\right)^{3 / 10} \\
& \approx 300\left|\cos \theta_{p}\right|^{3 / 5}
\end{aligned}
$$

which suggests that the echo protocol is very efficient also in $2 \mathrm{D}$.

\section{Appendix B: Density of thermal strongly interacting TLSs}

To estimate the typical distance $R_{T}$ between the probed TLS and its nearest thermal $S$-TLS, we use the relations [cf. Eqs. (24)]

$$
\begin{aligned}
& R_{T, 3 D}^{3} \int_{0}^{T} d E n_{S}(E)=1 \quad(3 \mathrm{D}), \\
& R_{T, 2 D}^{2} d \int_{0}^{T} d E n_{S}(E)=1 \quad(2 \mathrm{D}),
\end{aligned}
$$

where $n_{S}(E)$ is the DOS of $S$-TLSs. For $E<J_{0}^{\tau S}$, the interaction between the TLSs [Eqs. (39) and (40)] gives rise to a power-low pseudo-gap in the DOS of $S$-TLSs, namely $n_{S}(E) \propto E^{\mu}, \underline{23,39,41}$ Moreover, the DOS of $S$-TLSs is related to that of $\tau$-TLSs via $n_{S}(E)=g^{2} n_{\tau}(E)$ at $E=T_{U}$, where $T_{U} \approx 1 \mathrm{~K}$ is the temperature below which universality in amorphous and disordered systems is observed ${ }^{23}$ Since the DOS of $\tau$-TLSs is assumed to be constant, $n_{\tau}(E) \approx P_{0} \xi$, it follows that $n_{S}(E) \approx g^{2} P_{0} \xi\left(E / T_{U}\right)^{\mu}$. Using Eqs. (B1), we readily obtain

$$
\begin{aligned}
R_{T, 3 D}^{3} & =R_{T, 2 D}^{2} d=(\mu+1) T_{U}^{\mu} /\left(g^{2} P_{0} \xi T^{\mu+1}\right) \\
& =\frac{\mu+1}{g^{2} C_{0} \xi}\left(\frac{T_{U}}{T}\right)^{\mu} \frac{J_{0}^{\tau \tau}}{T} R_{0}^{3} .
\end{aligned}
$$

Setting $T \approx 35 \mathrm{mK}, T_{U} \approx 1 \mathrm{~K}$ and $J_{0}^{\tau \tau} \approx 1 \mathrm{~K}$, we obtain $R_{T, 2 D} \approx 10^{3} R_{0}$ (we note that this result is weakly dependent on $\mu$ ). Assuming $R_{0} \approx 1-2 \mathrm{~nm}$ we find $R_{T, 2 D} \sim 1 \mu \mathrm{m}$, somewhat larger than the sample size. For completeness we give here the typical coupling at this distance. Inserting Eq. (B2) in the interaction coefficients (40), we find the typical coupling strength between the probed TLS and its nearestc thermal $S$-TLS,

$$
\begin{aligned}
& J_{T}^{\tau S}=\frac{g}{\mu+1}\left(\frac{T}{T_{U}}\right)^{\mu} J_{T, 3 D}^{\tau \tau} \sim 2 \pi \cdot 10 g \mathrm{MHz} \quad(3 \mathrm{D}), \\
& J_{T}^{\tau S}=g^{2}\left[\frac{T^{\mu}}{(\mu+1) T_{U}^{\mu}}\right]^{3 / 2} J_{T, 2 D}^{\tau \tau} \sim 2 \pi \cdot g^{2} \mathrm{MHz} \quad(2 \mathrm{D}),
\end{aligned}
$$

where the coupling strength between the probed TLS and its nearest thermal $\tau$-TLS, $J_{T}^{\tau \tau}$, is defined in Eqs. (25) for both $D=3$ and $D=2$.
1 R. C. Zeller and R. O. Pohl, Phys. Rev. B 4, 2029 (1971).

2 J. F. Berret and M. Meissner, Z. Phys. B: Condens. Matter 70, 65 (1988).

3 R. O. Pohl, X. Liu, and E. Thompson, Rev. Mod. Phys. 74, 991 (2002).

4 P. W. Anderson, B. I. Halperin, and C. M. Varma, Philos. Mag. 25, 1 (1972).

5 W. A. Phillips, J. Low-Temp. Phys. 7, 351 (1972).

${ }^{6}$ W. A. Phillips, Rep. Prog. Phys. 50, 1657 (1987).

7 J. L. Black and B. I. Halperin, Phys. Rev. B 16, 2879 (1977).

8 A. L. Burin, D. Natelson, D. D. Osheroff, and Y. Kagan, in Tunneling Systems in Amorphous and Crystalline Solids, edited by P. Esquinazi (Springer, Berlin, 1998).
9 M. Schechter and P. C. E. Stamp, J. Phys. Condens. Matter 20, 244136 (2008).

10 E. Paladino, Y. M. Galperin, G. Falci, and B. L. Altshuler, Rev. Mod. Phys. 86, 361 (2014).

11 R. W. Simmonds, K. M. Lang, D. A. Hite, S. Nam, D. P. Pappas, and J. M. Martinis, Phys. Rev. Lett. 93, 077003 (2004).

12 J. M. Martinis, K. B. Cooper, R. McDermott, M. Steffen, M. Ansmann, K. D. Osborn, K. Cicak, S. Oh, D. P. Pappas, R. W. Simmonds, and C. C. Yu, Phys. Rev. Lett. 95, 210503 (2005).

13 M. Neeley, M. Ansmann, R. C. Bialczak, M. Hofheinz, N. Katz, E. Lucero, A. OConnell, H. Wang, A. N. Cleland, and J. M. Martinis, Nature Phys. 4, 523 (2008). 
14 Y. Shalibo, Y. Rofe, D. Shwa, F. Zeides, M. Neeley, J. M. Martinis, and N. Katz, Phys. Rev. Lett. 105, 177001 (2010).

15 J. Lisenfeld, C. Müller, J. H. Cole, P. Bushev, A. Lukashenko, A. Shnirman, and A. V. Ustinov, Phys. Rev. Lett. 105, 230504 (2010).

16 As mentioned above, the strain field in the absence of any external perturbation is also responsible for the relaxation of the probed TLS, discussed in Ref. 18, and for the TLSTLS interactions [see Eqs. (2)-(4) and the third term of Eq. (6)]. In Eq. (5) we focus only on the applied strain $\epsilon_{p}$, whose sole effect is to externally tune the energy bias of the probed TLS.

17 G. J. Grabovskij, T. Peichl, J. Lisenfeld, G. Weiss, and A. V. Ustinov, Science 338, 232 (2012).

18 J. Lisenfeld, A. Bilmes, S. Matityahu, S. Zanker, M. Marthaler, M. Schechter, G. Schön, A. Shnirman, G. Weiss, and A.V. Ustinov, arXiv:1601.03213

19 We set $\hbar=k_{B}=1$ and measure energies in units of frequencies or temperatures.

20 W. Arnold and S. Hunklinger, Solid State Commun. 17, 833 (1975).

21 W. Arnold, C. Martinon, and S. Hunklinger, J. Physique Colloq. 39, C6-961 (1978).

22 An alternative explanation was given in S. Zanker, M. Marthaler, G. Schön, arXiv:1601.03861

23 M. Schechter and P. C. E. Stamp, Phys. Rev. B 88, 174202 (2013).

24 E. Paladino, L. Faoro, G. Falci, and R. Fazio, Phys. Rev. Lett. 88, 228304, (2002).

25 Y. M. Galperin, B. L. Altshuler, and D. V. Shantsev, in Fundamental Problems of Mesoscopic Physics, edited by I. V. Lerner et. al. (Kluwer, Dordrecht, 2004); cond-mat/0312490.

26 J. Bergli, Y. M. Galperin, and B. L. Altshuler, New. J. Phys. 11, 025002 (2009).

27 J. Jäckle, Z. Phys. 257, 212 (1972).

28 A. L. Burin and Yu. Kagan, JETP 79, 347 (1994).

${ }^{29}$ Y. M. Galperin, B. L. Altshuler, J. Bergli, and D. V. Shantsev, Phys. Rev. Lett. 96, 097009 (2006).

30 G. Ithier, E. Collin, P. Joyez, P. J. Meeson, D. Vion, D. Esteve, F. Chiarello, A. Shnirman, Y. Makhlin, J. Schriefl, and G. Schön, Phys. Rev. B 72, 134519 (2005)

31 J. Schriefl, Y. Makhlin, A. Shnirman, and G. Schön, New J. Phys. 8, 1 (2006).

32 S. Hunklinger, Cryogenics 28, 224 (1988).

33 J. Lisenfeld, private communication.

${ }^{34}$ We use the term "rate" to refer to the inverse of the time for which the signal has decayed to $e^{-1}$. The decay, however, might not be an exponential one.

35 J. Burnett, L. Faoro, I. Wisby, V. L. Gurtovoi, A. V. Chernykh, G. M. Mikhailov, V. A. Tulin, R. Shaikhaidarov, V. Antonov, P. J. Meeson, A. Y. Tzalenchuk, and T. Lindström, Nat. Commun. 5, 4119 (2014).

${ }^{36}$ L. Faoro and L. B. Ioffe, Phys. Rev. B 91, 014201 (2015).

37 A. Churkin, S. Matityahu, A. L. Burin, and M. Schechter, to be published.

38 A. Gaita-Ariño and M. Schechter, Phys. Rev. Lett. 107, 105504 (2011).

39 A. Churkin, D. Barash, and M. Schechter, J. Phys. Condens. Matter 26, 325401 (2014).

40 The low-energy effective Hamiltonian includes also a random field term of the form $\sum_{i}\left(h_{i}^{\tau} \hat{\tau}_{i}^{z}+h_{i}^{S} \hat{S}_{i}^{z}\right)$, where the random fields $h^{\tau}$ and $h^{S}$ have typical strengths $h^{\tau} \lesssim g J_{0}$ and $h^{S} \lesssim J_{0}$. Such a term has no effect on the dynamics of the TLSs and can be absorbed into the energy bias $\Delta$.

41 A. Churkin, D. Barash and M. Schechter, Phys. Rev. B 89, 104202 (2014).

42 A. Churkin, I. Gabdank, A. Burin, and M. Schechter, arXiv:1307.0868

43 L. Faoro and L. B. Ioffe, Phys. Rev. Lett. 109, 157005 (2012).

44 This condition is equivalent to $\tau_{c} \lesssim T_{\varphi, E / R}$ where $\tau_{c}$ is the time over which the correlation function $S_{X}(t)$ decays.

45 A. P. Paz, I. V. Lebedeva, I. V. Tokatly, and A. Rubio, Phys. Rev. B 90, 224202 (2014).

${ }^{46}$ M. S. Khalil, S. Gladchenko, M. J. A. Stoutimore, F. C. Wellstood, A. L. Burin, K. D. Osborn, Phys. Rev. B 90, 100201(R) (2014).

47 A. L. Burin, S. Matityahu, and M. Schechter, Phys. Rev. B 92, 174201 (2015) 